\title{
Kinetics of the Radicals Induced in Gamma Irradiated Sulfafurazole: An EPR Study
}

\author{
Şeyda Çolak and Mustafa Korkmaz \\ Physics Engineering Department, Hacettepe University, 06532 Beytepe, Ankara, Turkey. \\ Reprint requests to Prof. M. K.; Fax: +90-312-299-20-37; E-mail: seyda@hacettepe.edu.tr
}

Z. Naturforsch. 59a, 481 - 487 (2004); received April 1, 2004

The spectroscopic and kinetic features of the radiolytic intermediates produced in gamma irradiated sulfafurazole (SFZ) were investigated at different temperatures in the dose range 5-50 kGy using EPR and IR techniques. The imodiation produced two species (A, B) in SFZ. The heights of the peaks were used to monitor the temperature, time dependent and kinetic features of the radical species contributing to the EPR spectrum. The applicability of EPR technique for monitoring radiosterilization of SFZ is discussed. The radiation yield of solid SFZ was found to be very low $(\mathrm{G}=0.16)$, and basing on this it was concluded that SFZ and SFZ containing drugs can be safely sterilized by radiation. The EPR data were used to characterize the contributing radicals produced in gamma irradiated SFZ. No definite difference was observed between unirradiated and irradiated IR spectra of SFZ.

Key words: Radiosterilization; Sulfafurazole; Radical Kinetics; EPR; IR.

\section{Introduction}

Sterilization of medical products is one of the applications of high energy radiation $[1-3]$. This technique is succesfully used for sterilizing solid pharmaceutical compounds which are otherwise sensitive to traditional methods of sterilization employing heat and ethylene oxide gas [4-7]. The advantages of sterilization by irradiation include high penetrating power, low chemical reactivity, low measurable residues, small temperature rise and the fact that there are fewer variables to control. Thus, the sterilization can be carried out on packaged products [8-10]. Nevertheless, beside its advantages, radiosterilization also has some drawbacks. The radiosensitivity of irradiated pharmaceuticals is especially important because radiation can cause a decrease in the amount of active drug by destroying it and creating reactive molecular fragments $[4,11,12]$ which may result in a toxicological hazard. Thus, the study of radiation induced radicals and chemical products in drugs is necessary, both to determine the feasibility of the radiation treatment and to control it.

Electron Paramagnetic Resonance (EPR) technique appears to be suited for the determination of rare free radicals in complex media and could permit the elucidation of the mechanism of radiolysis even with small radiation doses. EPR measurements can also be used to detect and to distinguish irradiated drugs from unirradiated ones [11,13,14].

Sulfonamides are used as active ingradients in different drugs to treat infections caused by bacteria. SFZ is one of the most commonly used sulfonamides for this type of treatments. Therefore, the aims of the present work are: to investigate the radiation sensitivity of solid SFZ in the dose range of 5-50 kGy and to explore the potential use of EPR technique in monitoring the radiosterilization of SFZ and/or drug delivery system containing SFZ as active ingredient, through a kinetic study of the radiation induced radicals in gamma irradiated SFZ by EPR spectroscopy.

\section{Materials and Methods}

Sulfafurazole (SFZ), with its chemical name 4-amino-N-(3,4-dimethylisoxazol-5-yl)benzenesulfonamide, of spectroscopic grade was provided from the Faculty of Pharmacy of the Hacettepe University (Ankara) and was stored at room temperature in a closed container protected from light. No further purification was performed. SFZ is a white or yellowish white odourless crystalline powder which melts about $197{ }^{\circ} \mathrm{C}$ with decomposition. Its molecular structure is given in Figure 1. Irradiations were performed at room temperature and at $77 \mathrm{~K}$ in dark using a ${ }^{60} \mathrm{Co}$ gamma 


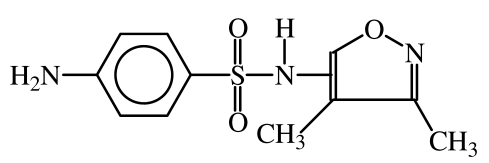

Fig. 1. Molecular structure of sulfafurazole.

cell supplying a dose rate of $2.52 \mathrm{kGy} / \mathrm{h}$ as an ionizing radiation source at the Sarayköy Establishment of the Turkish Atomic Energy Agency in Ankara. The dose rate was measured by a Fricke dosimeter. Investigations were performed on samples irradiated at four different doses $(5,10,25$, and $50 \mathrm{kGy})$.

The EPR measurements were carried out using a Varian 9"-E line-X band EPR spectrometer operating at $9.5 \mathrm{GHz}$ and equipped with a $\mathrm{TE}_{104}$ rectangular double cavity containing a standard sample (DPPH) in the rear resonator which remained untouched throughout the experiment. Signal intensities were calculated from the first derivative spectra and compared with that obtained for a standard sample (DPPH) under the same spectrometer operating conditions. The sample temperature inside the microwave cavity was monitored with a digital temperature control system (Bruker ER 4111-VT). The latter provided the opportunity of measuring the temperature with an accuracy of $\pm 0.5 \mathrm{~K}$ at the site of the sample. A cooling, heating and subsequent cooling cycle was adopted to monitor the evolution of the EPR line shape with temperature, using samples irradiated at room temperature. The latter was first decreased to $120 \mathrm{~K}$ starting from room temperature, then increased to $400 \mathrm{~K}$ with an increment of $20 \mathrm{~K}$ and finally decreased again to room temperature. Variations in the line shape and signal intensities with microwave power were also studied in the range $0.5-$ $80 \mathrm{~mW}$ for samples irradiated at room temperature and at $77 \mathrm{~K}$.

Kinetic studies of the contributing free radicals were performed at different temperatures. To achieve this goal, the samples irradiated at room temperature were heated to predetermined temperatures $(320 \mathrm{~K}, 335 \mathrm{~K}$, $350 \mathrm{~K}, 358 \mathrm{~K}, 383 \mathrm{~K}, 393 \mathrm{~K}$, and $413 \mathrm{~K}$ ), kept at these temperatures for predetermined times $(3,5,8,10,14$, $20,25,30,35,40,45,55,60,65,80,90,120,150$, $160,200,210,220 \mathrm{~min}$ ) and then cooled to room temperature, and their EPR spectra were recorded. The results were the average of five replicates for each radiation dose. A simulation calculation based on a model of two species (anticipating the results of our studies), was performed to determine the spectroscopic features of the contributing free radicals.

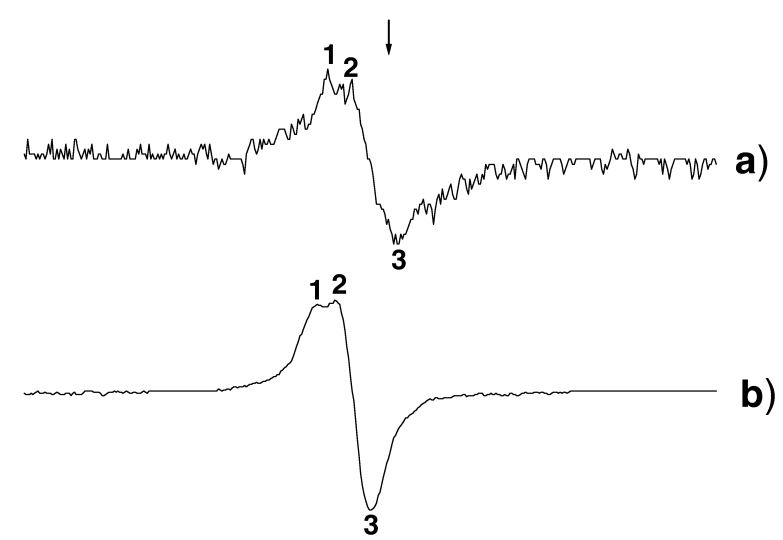

Fig. 2. Room temperature EPR spectra of gamma irradiated SFZ with assigned peak numbers. a) $5 \mathrm{kGy}$, b) $50 \mathrm{kGy}$. Arrow indicates the position of DPPH line.

\section{Experimental Results and Discussion}

\subsection{General Features of the EPR Spectra}

Unirradiated SFZ exhibited no EPR signal. Samples irradiated at $77 \mathrm{~K}$ and at room temperature showed a very simple EPR spectrum consisting of three resonance peaks appearing at $g$ values of 2.0081 (peak 1), 2.0067 (peak 2), 2.0034 (peak 3) as given in Fig. 2a and $b$ with their assigned peak numbers. Increase in the absorbed dose did not create any pattern change in the spectra of the samples irradiated at $77 \mathrm{~K}$ and at room temperature. It was concluded that the irradiation temperature was not an important parameter for the formation of radiation induced radical species in irradiated SFZ. Consequently, all further studies were performed on samples irradiated at room temperature.

Variations of the height, which was measured with respect to the base line and normalized to the receiver gain, the mass of the sample and the intensity of the standart, of these resonance peaks with an applied microwave power in the range $0.5-80 \mathrm{~mW}$ were studied first. The results of these studies indicated that peak heights saturate as inhomogeneously broadened resonance lines and that they saturate faster at low temperature $(120 \mathrm{~K})$. Theoretical functions, best fitting to microwave saturation data derived from $120 \mathrm{~K}$ and room temperature spectra, were calculated assuming two exponentially growing curves of different characteristics associated with two radical species involved in the formation of low and room temperature experimental EPR spectra. From the results of these calculations it was concluded that two different radical species were 


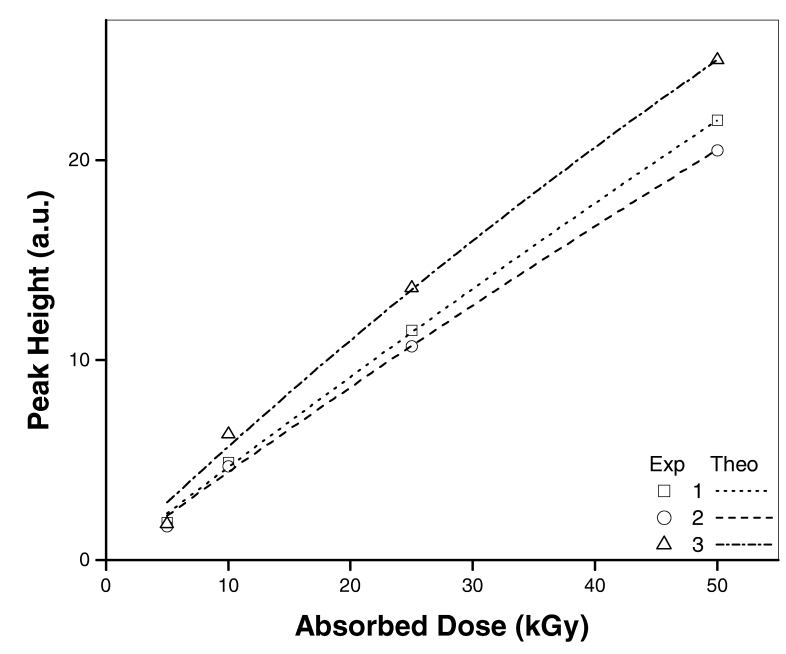

Fig. 3. Variation of peak heights with absorbed radiation dose. $\square$ (peak 1), $\bigcirc$ (peak 2), $\triangle$ (peak 3).

produced in gamma irradiated SFZ, and that one of the species (assigned as B) saturates more easily than the other one (assigned as A) at low $(120 \mathrm{~K})$ and room temperatures.

\subsection{Dose-Response Curves}

A radiation sensitivity of a substance or mixture is expressed by a $G$ value, that is, by the number of radicals produced by the absorbed radiation dose per $100 \mathrm{eV}$. A higher concentration of radicals, generated at the same absorbed dose of radiation, indicates a higher sensitivity of the substance toward the type of radiation used. Variations of the heigths of the resonance peaks assigned as 1,2, and 3 with absorbed gamma radiation dose were found to follow the function given in by

$$
I=I_{\mathrm{o}}\left(1-e^{-a^{*} D}\right)
$$

in the dose range of $0-50 \mathrm{kGy}$ (Fig. 3) for SFZ. $D$ stands for the absorbed dose in kGy and $a^{*}$ for radiation dose growth parameter. The latter were found to have the values $0.0028,0.0036$, and $0.0065\left(\mathrm{kGy}^{-1}\right)$ for the peaks 1,2 , and 3 , respectively.

\subsection{Stabilities of the Radical Species}

The stability of radicals in an irradiated drug is as important as the radiosensitivity of these materials. Therefore, this feature was also studied. Samples irradiated at different radiation doses were used to achieve this goal. The decay rates of the samples irradiated at different doses and stored at normal and stability conditions $\left(75 \%\right.$ relative humidity, $40{ }^{\circ} \mathrm{C}$ ) were found to be independent of the irradiation dose. Thus, the signal intensity decay data obtained for a sample irradiated at a dose of $50 \mathrm{kGy}$ were used to get the decay characteristics of the contributing species under both storing conditions. The data relative to the variations of the signal heights of the resonance peaks over a period of 90 days for samples stored at room temperature and stability condition data were found best fitt the sum of two exponentially decaying functions

$$
I=I_{\mathrm{Ao}} e^{-k_{\mathrm{A}} t}+I_{\mathrm{Bo}} e^{-k_{\mathrm{B}} t} .
$$

In (2) $t ; I_{\mathrm{Ao}}, I_{\mathrm{Bo}} ; k_{\mathrm{A}}$ and $k_{\mathrm{B}}$ stand for the time elapsed after the stopping of irradiation, the initial signal intensities and decay constants, respectively. This result, which agrees with the microwave saturation data, was considered, once more, as the justification of the presence of two species of fairly different decay characteristics contributing to the formation of the EPR spectra of irradiated SFZ. The following decay constants were obtained for the radical species $\mathrm{A}$ and $\mathrm{B}$ at normal $\left[k_{\mathrm{A}}=552 \pm 56 \cdot 10^{-5} \mathrm{day}^{-1} ; k_{\mathrm{B}}=1228 \pm\right.$ $110 \cdot 10^{-5}$ day $\left.^{-1}\right]$ and stability $\left[k_{\mathrm{A}}=4031 \pm 280\right.$. $10^{-5}$ day $^{-1} ; k_{\mathrm{B}}=7669 \pm 480 \cdot 10^{-5}$ day $\left.^{-1}\right]$ conditions. As expected, the decays of the contributing species were faster under stability conditions. However species B was found to decay faster than the radical A under normal and stability conditions.

\subsection{Variation of the Peak Heights with Temperature}

A variable temperature study of the peak heights in the range of $295-120 \mathrm{~K}$ and $295-400 \mathrm{~K}$ was also performed. Cooling the sample to room temperature did not create any pattern change in the spectra except slight reversible increases in the peak heights, likely due to classical paramagnetic behaviours of the contributing species. Warming the sample above room temperature produced practically no changes in the heights of all observed peaks up to $320 \mathrm{~K}$. However, increase of the sample temperature above $320 \mathrm{~K}$ caused continuous decreases of all resonance peaks up to $360 \mathrm{~K}$. Then relatively sharp decreases in the heights occurred (Fig. 4). The observed signal intensity decreases above room temperature of all resonance peaks were found to be irreversible, orginating from relatively high decays of the contributing species at high temperatures. This incited us to perform anneal- 


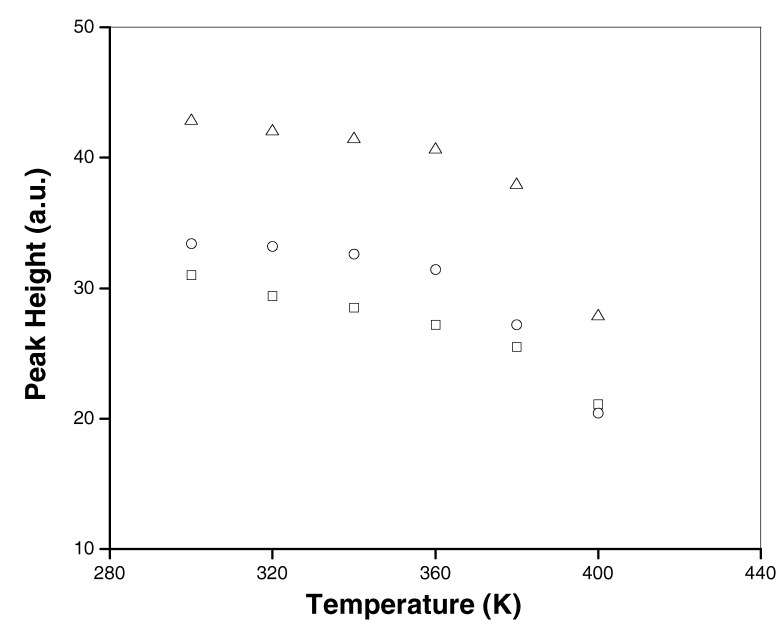

Fig. 4. Variation of the peak heights with temperature in the range of $295-400 \mathrm{~K}, \square$ (peak 1$), \bigcirc$ (peak 2), $\triangle$ (peak 3 ).

ing studies on irradiated SFZ to determine the decay constant at high temperatures, and thus, to calculate the activation energies relevant to the radical decay processes.

\subsection{Decays of Species in Annealed Samples}

Radical decay rates depend on the nature of the matrix containing radicals, and annealing is a constant process with local diffusion of radicals and molecules in some softening of defects or irregularities [15]. At room temperature the decay is very slow, and many radical-molecule reactions observed in the liquid state are not observed in the solid state. Two species responsible for the EPR spectrum of irradiated SFZ are expected to have different decay characteristics depending on the sample temperature. In other words, the decay rates of the species at high temperature are expected to be higher than those at low temperatures. In fact, it was found that this is the case. Increase in temperature accelerated the decays of the species. Signal intensity decay results obtained for peak 3 of an SFZ sample irradiated with $50 \mathrm{kGy}$ and annealed at different temperatures for different times are given in Figure 5. Similar decay result were obtained for peak 1 and 2. Experimental peak height decay data obtained for samples annealed at different temperatures were used to calculate the decay constants of the contributing species at the annealing temperatures, assuming that the species follow first order kinetics as given by (2). Two species of different decay constants decaying with assumed kinetics were found to fit best the experimental signal intensity decay data.

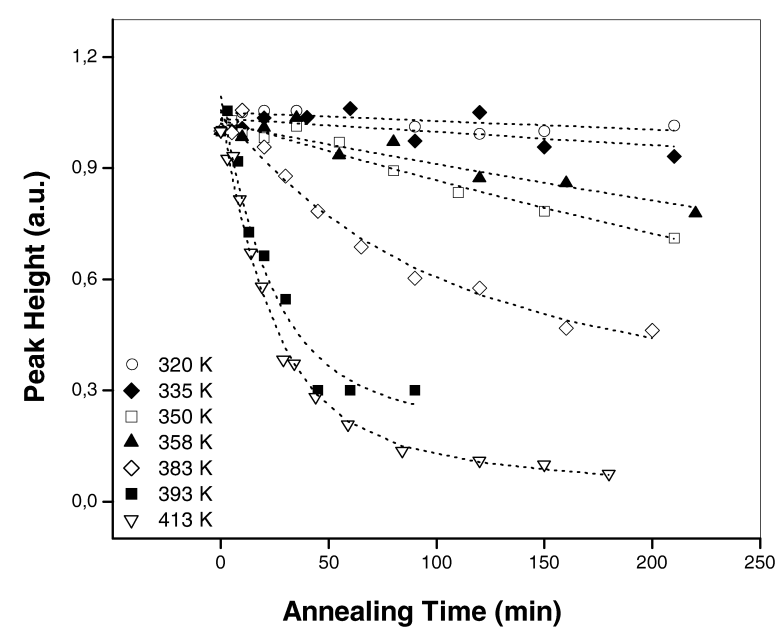

Fig. 5. Signal intensity decay results for peak 3 of samples irradiated at a dose of $50 \mathrm{kGy}$ and annealed at seven different temperatures.

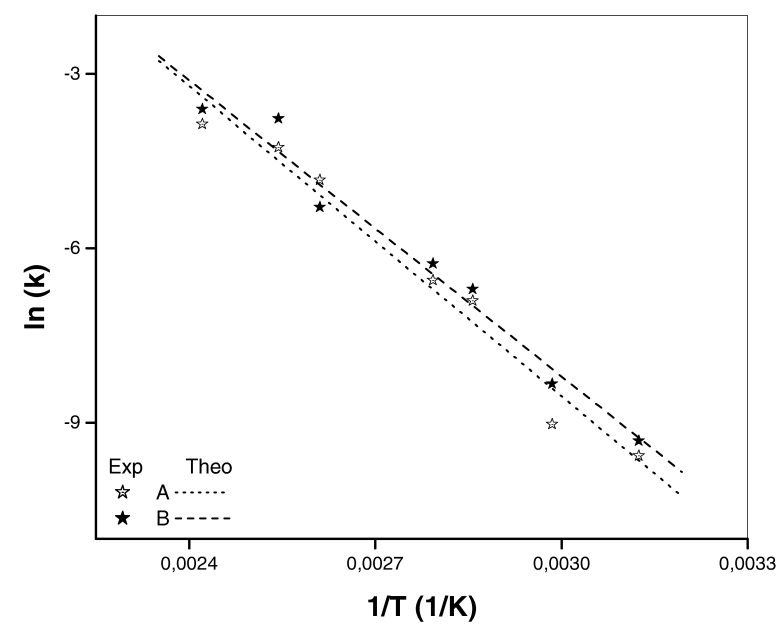

Fig. 6. Arrhenius plot constructed for an SFZ sample irradiated at a dose of $50 \mathrm{kGy}$. Symbols: experimental, dashed lines: theoretical.

Dotted lines in Fig. 5 show the theoretical curves obtained by this procedure. Arrhenius plots were also constructed to determine the activation energies of the contributing species, see Figure 6. The activation energies were found to be $E_{\mathrm{A}}=(73.7 \pm 5.9) \mathrm{kJ} / \mathrm{mol}$ and $E_{\mathrm{B}}=(70.3 \pm 5.4) \mathrm{kJ} / \mathrm{mol}$ for species $\mathrm{A}$ and $\mathrm{B}$, respectively.

\subsection{Proposed Radical Species and Spectrum Simulation}

Excited molecules are produced both directly and through radical-cation-neutralization [16]. They may 
Table 1. Calculated spectroscopic parameters for contributing radical species.

\begin{tabular}{|c|c|c|c|}
\hline \multirow[b]{2}{*}{ Radical Species } & \multirow[b]{2}{*}{$g$ factor } & \multicolumn{2}{|c|}{ Spectroscopic Parameters } \\
\hline & & Line Width $(\mathrm{mT})$ & Relative Weight \\
\hline \multirow[t]{4}{*}{$\left(\mathrm{SO}_{2}\right)^{-}(\mathrm{A})$} & $\begin{array}{c}2.0042 \\
( \pm 0.0002)\end{array}$ & $\begin{array}{c}0.5 .1 \\
( \pm 0.020)\end{array}$ & 0.52 \\
\hline & $\begin{array}{c}2.0090 \\
( \pm 0.0002)\end{array}$ & & \\
\hline & & $\begin{array}{c}0.178 \\
( \pm 0.002)\end{array}$ & 0.48 \\
\hline & $\begin{array}{c}2.0047 \\
( \pm 0.0002)\end{array}$ & & \\
\hline
\end{tabular}

decompose to radicals by rupture of chemical bonds. However, radicals which are molecular fragments can not diffuse in solid matrices due to cage effects, and therefore immediate germinate termination reactions are possible [15]. Excited molecules and, as a result, radicals are localized along the track in regions of high local concentration. $\mathrm{SO}_{2}$ is the most sensitive group to radiation in SFZ molecules due to its high electrophilicity. Therefore radicals with unpaired electrons, localised on $\mathrm{SO}_{2}$ groups, are expected to be likely produced on irradiation of SFZ, as in the case of sulphur containing compounds [17-19]. As in the present work, these radicals do not exhibit hyperfine structure, but they have a $g$ tensor of orthorombic symmetry with an average value varying between 2.0037-2.0059 [17,18]. Experimental $g$ values determined in the present work for resonance peaks of the gamma irradiated SFZ $\left(g_{1}=2.0089, g_{2}=2.0060\right.$, $g_{3}=2.0038$ for peaks 1,2 , and 3 , respectively) fall into this range. Consequently, we believe that $\mathrm{SO}_{2}{ }^{-}$ ionic radicals (hereafter radical $\mathrm{A}$ ) and neutral species $[\mathrm{O}=\mathrm{S}=\mathrm{O}]^{*}$ (hereafter $\mathrm{B}$ ) are the responsible magnetic units from the three EPR peaks of gamma irradiated SFZ. A and B, produced in irradiated crystalline powder of SFZ, are randomly oriented. However the motion of $\mathrm{B}$ is restricted due to the big group attached to it, so that it gives rise to powder spectra with principal $g$ values varing between $g_{x x}=2.0022-2.0031$, $g_{y y}=2.0015-2.0098$, and $g_{z z}=2.0058-2.0066$ [18]. As for the $\mathrm{SO}_{2}-$ ionic radical or radical $\mathrm{A}$, its motional freedom is high due to very weak steric effect experienced by this radical. As a result of this, it gives rise to a single resonance line of an average spectroscopic factor varying between $2.0037-2.0059$ [17, 18].

Simulation calculations were performed to support the idea put forward with the species responsible for the observed experimental three peaks EPR spectra of gamma irradiated SFZ, and to determine correct spec-

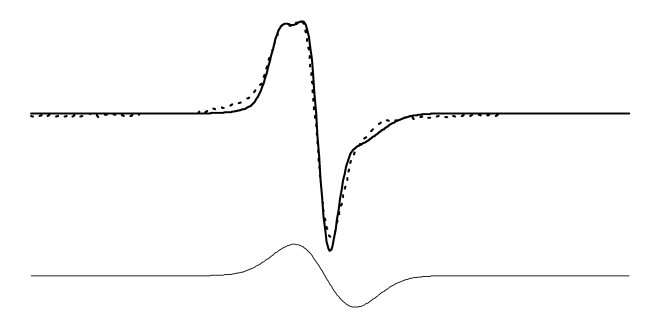

c)

a)

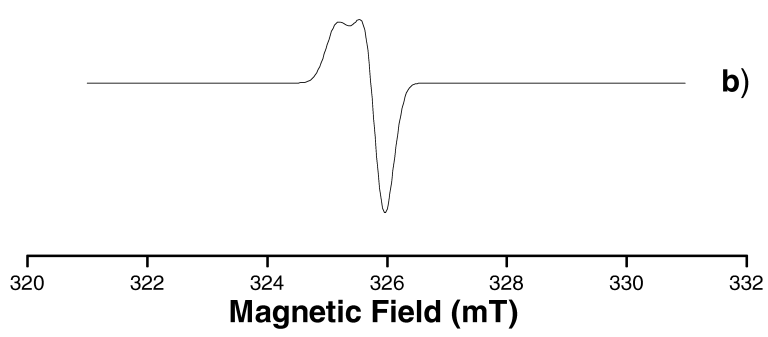

Fig. 7. Experimental and calculated EPR spectra for SFZ irradiated at a dose of $50 \mathrm{kGy}$. a), b): calculated spectra for radical species $\mathrm{A}$ and $\mathrm{B}$, respectively. c): experimental and calculated sum spectra. Solid line: theoretical, dashed line: experimental.

troscopic parametes of the contributing species, assuming the presence of $\mathrm{A}$ and $\mathrm{B}$, exhibiting isotropic and axially symmetric $g$ tensors. Room temperature experimental signal intensity data obtained for a sample irradiated with a dose of $50 \mathrm{kGy}$ were used as input to carry out the simulation calculations. The results of these calculations are summarized in Table 1, and theoretical spectra derived from the parameters given in this table are presented in Fig. 7 with their experimental counterpart. The spectra of the contributing species $(\mathrm{A}, \mathrm{B})$ are also given in Fig. 7. The line width of neutral species B is small compared with ionic radical A, but both species equally dominate the EPR spectrum. As seen, the agreement between the experimental and theoretical spectra is fairly good, which indicates that the model based on two species of different characteristic features explains well the experimental EPR spectra of gamma irradiated SFZ.

Gamma irradiated frozen aqueous solutions of sulphacetamide have been examined in [20], using EPR to look for possible intermediates in the radiation degradative pathway. The examination has been conducted at $77 \mathrm{~K}$, and a single resonance line centered at $g=2.0075$ was reported for a sample annealed to $120 \mathrm{~K}$. This value falls into the $g$ value range reported in the present work for gamma irradiated solid SFZ (Table 1). 


\subsection{FT- IR Spectra of SFZ}

IR spectroscopy was thought to be a complementary technique to elucidate the types and structures of the radiation induced species, and FT-IR spectra of both unirradiated and gamma irradiated SFZ samples were also recorded at room temperature. However, significant differences between these spectra, attributable to any intermediates created by radiation, were not observed. This was considered to be likely because the amounts of the radiation induced intermediates were very small. This result was considered as correlating with the relatively small $G$ value (0.16) reported in the present work for gamma irradiated SFZ by EPR technique.

\section{Conclusions}

Samples of SFZ, irradiated at $77 \mathrm{~K}$ and at room temperature, exhibited very simple EPR spectra consisting of three resonance peaks which saturated as inhomogenously brodened resonance lines in the studied microwave power range $(0.5-80 \mathrm{~mW})$. However, the radiation yield of SFZ was very small $(G=0.16)$ compared with those reported for sulfonamide solutions ( $G$ varies in the range of 3.5-5.1), but it falls into the range of the $G$ values reported for solid sulfonamides $(0.15-0.6)$ [20,21]. This difference in $G$ values was believed to originate from hyrated electrons $\left(\mathrm{e}^{-}\right.$eq $)$and hydroxyl radicals $(\cdot \mathrm{OH})$ produced in large amounts as radiolytic intermediates in irradiated aqueous solutions of sulfonamides. Although, the sensitivity of SFZ to gamma radiation was not high, the detection and discrimination of unirradiated SFZ from irradiated one turned out to be possible even at low radiation doses (Fig. 2a). Most sensitive subgroup of the SFZ molecule to radiation, that is $\mathrm{SO}_{2}$, was believed to be at the origin of the observed EPR spectra of irradiated SFZ. Therefore, tentative ionic radicals $\left(\mathrm{SO}_{2}\right)(\mathrm{A})$ and neutral species $[\mathrm{O}=\mathrm{S}=\mathrm{O}]^{*}(\mathrm{~B})$ were assumed to

[1] G. P. Jacobs, Radiat. Phys. Chem. 26, 133 (1985).

[2] W. Wogl, Radiat. Phys. Chem. 25, 425 (1985).

[3] L. Varshney and K. M. Patel, Radiat. Phys. and Chem. 43, 471 (1994).

[4] C. Boess and K. W. Bögl, Drug Dev. Ind. Pharm. 22, 495 (1996).

[5] E. R.L. Gaughran and A.J. Goudie, Sterilization by ionizing radiation: sterilization of medical products be produced upon irradiation, giving rise to isotropic and axially symmetric EPR spectra, respectively. Resonance signals originating from $\mathrm{A}$ and $\mathrm{B}$ equally dominate the EPR spectrum. A model based on the proposed tentative species, that is two species exhibiting isotropic and axially symmetric $g$ tensors, was found to describe best the experimental results obtained from dose-response, decays of radicals in normal and stability conditions, and thermal studies.

Both species were not stable at normal and at stability conditions and, especially, at high temperatures (Figs. 4 and 5). However, decay data obtained for samples, stored over a period of 90 days at normal and stability conditions, indicated that the EPR signal could be detected even after a storage period of several months in irradiated SFZ. Thus it was concluded that irradiated SFZ could be distinguished from unirradiated one even long after the irradiation by EPR spectroscopy. At stability conditions $\left(40{ }^{\circ} \mathrm{C}\right.$ and $75 \%$ humidity) the decay constants of the participating species were observed to increase several times. This last result was considered as a positive point in diminishing the amount of the radiolytic intermediates in irradiated SFZ. The activation energies relevant to transformation reactions at high temperature of the species $\mathrm{A}$ and $\mathrm{B}$ to nonmagnetic units, that is the reaction of loosing their unpaired electrons, were observed to be very similar. This shows that the thermal behaviour of both species is not very different at high temperature.

Basing on the above results, it was concluded that EPR spectroscopy could be used as a potential technique in monitoring the radiosterilization of SFZ and/or drug delivery systems containg SFZ as active ingradient.

\section{Acknowledgements}

We thank Prof. Dr. Yekta Özer from Faculty of Pharmacy of Hacettepe University for providing SFZ of spectroscopic grade.

by ionizing radiation, Vol. II., Multiscience Publishers Ltd., Montreal, Quebeq, Canada, 1977.

[6] R. Pourahmad and R. Pakravan, Radiat. Phys. and Chem. 49, 285 (1997).

[7] B. D. Reid, J. Pharm. Sci. and Tech. 49, 2 (1995).

[8] N. Barbarin, B. Rollmann, and B. Tilquin, Int. J. Pharm. 178, 203 (1999). 
[9] J. P. Basly, I. Longy, and M. Bernard, Analytica Chimica Acta. 359, 107 (1998).

[10] M. Gibella, A. S. Crucq, B. Tilquin, P. Stocker, G. Lesgards, and J. Raffi, Radiat. Phys. Chem. 58, 69 (2000).

[11] T. Miyazaki, J. Arai, K. Kaneko, K. Yamamoto, M. Gibella, and B. Tilquin, J. Pharm., Sci. 83, 1643 (1994).

[12] R. H. Schuler, Radiat. Phys. and Chem. 43, 417 (1994).

[13] J. P. Basly, I. Basly, and M. Bernard, Int. J. Radiat. Biol. 75, 259 (1999).

[14] K. W. Bögl, Appl. Radiat. Isot. 40, 1203 (1989).

[15] B. Tilquin, Composant radicalaire des transformations radio-initieés dans les alcanes à $77 \mathrm{~K}$, thèse dagrégation, UCL, Ciaco-la-Neuve, Belgique, 1985.

[16] A. C. Dusaucy and B. Tilquin, Radiat. Phys. and Chem. 37, 217 (1991).

[17] M. I. Samoilovich and L. I. Tsinober, Sov. Phys. Crystallogr. 14, 656 (1970).

[18] R. Huzimura, Jap. J. Appl. Phys. 18, 2031 (1979).

[19] O. Katzenberger, R. Debuyst, P. De Canniere, F. Dejehet, D. Apers, and M. Barabas, Appl. Radiat. Isot. 40, 1113 (1989).

[20] G. O. Philips, D. M. Power, and M. C. G. Sewart, Radiat. Res. 46, 236 (1971).

[21] G. O. Philips, D. M. Power, and M.C.G. Sewart, Radiat. Res. 53, 204 (1973). 